\title{
Investigating the Mettapracharak (Watraikhing) vision rehabilitation outcome using the Thai version of the low vision Quality-of-Life Questionnaire (LVQOL)
}

\author{
Penpimol Yingyong* \\ Mettapracharak (Wat Raikhing) Hospital, Raikhing, Sampran, Nakhon Pathom 73210, Thailand
}

\begin{abstract}
Background: Quality of life is the outcome of the visual rehabilitation. Analysis of its effectiveness should have been comprehensively conducted to improve the remained visual function efficiency. Clinically meaningful changes are evaluated to predict the impact on the visual rehabilitation.

Objective: To determine the outcome of the Mettapracharak Vision Rehabilitation service unit by the Thai version of the Low Vision Quality-of-Life Questionnaire (LVQOL).

Methods: A prospective study of 125 participants (age between 12-88 years; 72 (57.6\%) females and 53 (42.4\%) males attending hospital-based low vision services completed the LVQOL questionnaire before and 3 months after their vision rehabilitation from October 2016 to March 2017 .Their visual acuities in the betterseeing eye after the best correction were worse than 20/70 and/or visual field less than 30 degree from the fixation. Comprehensive low vision examination, providing low vision aids and vision-based skill rehabilitation were administered to enhance their vision-related quality of life (functional abilities).

Results: Glaucoma was the most common cause of visual impairment. Almost all the patients reported better changes on the subscales of the questionnaires with statistically significant differences $(\mathrm{p}<0.05)$ compared between before and after vision rehabilitation. The magnitude and the clinical significance of the overall quality of life scores were found to be relatively moderate effect size and the standardized response mean value.

Conclusion: This study was to administer a better understanding of the vision rehabilitation services with effectively clinical evidences. Although these findings generated many implementations for the future researches on the vision rehabilitation strategies, more holistic approaches should have been challenging to be evaluated.
\end{abstract}

\section{Introduction}

The visual impairment is principally chronic disease such as uncorrected refractive errors, cataracts and glaucoma respectively [1]. Its prevalence has been dramatically increasing due to long-life expectancy and the better medical care. By the year 2010, the World health organization estimated the amount of the visual impairment groups for all ages about 285 million people and the visual disabilities, the aging-related impairments, are commonly found in old persons more than 50 years old [1]. The Thai national survey of blindness and low vision showed the increasing rate of blindness from $0.31 \%$ [2] to $0.59 \%$ [3] and the increasing rate of low vision $0.59 \%$ [2] to $1.57 \%$ [3]. Low vision rehabilitation maximized the residual seeing ability by training the daily living activity. Therefore, the effectiveness in low vision rehabilitation should have been conducted to provide the clinically meaningful change results after the appropriate rehabilitation. To our knowledge, there are few reports about the effective outcome of the low vision rehabilitation outcome in Thailand. Some studies were conducted to evaluate the outcome improvements in term of the quality of life after the visual rehabilitation intervention [4-6]. The Thai, low vision quality-of-life questionnaire (LVQOL) [7] was developed to measure the Thai visual impairment quality of life with good construct validity (Factor loading $>0.7$ ) and the high degree of reliability (Internal consistency between 0.7224-0.9099). The aim of this study was to determine the outcome of the Mettapracharak Vision Rehabilitation service unit by the LVQOL [7].

\section{Materials and methods}

The present study was a prospective study of the quality of life record (LVQOL) scores of the 125 outpatients before and 3 months after visual rehabilitation in the Low vision unit at the Mettapracharak(Watraikhing) Hospital. It was conducted from October 2016 to March 2017. The enrollment of the participants was approved by the Mettapracharak Hospital, Nakhonpathom research ethics committee to carry out the study before its commencement. The research protocol adhered to the tenets of the Declaration of Helsinki. The inclusion criteria of the participants included the participants with the visual acuities in the better-seeing eye after the best correction were worse than 20/70 and/or visual field less than 30 degree from the fixation that regular eyeglasses, medicine or surgery cannot improve the criteria condition. Exclusion criteria were neurologic diseases that limited the activities of daily living, severe cognitive impairment that

Correspondence to: Penpimol Yingyong, Mettapracharak (Wat Raikhing) Hospital, Raikhing, Sampran, Nakhon Pathom 73210, Thailand. E-mail: penpimol1960@gmail.com

Key words: Mettapracharak(Watraikhing), Vision Rehabilitation, Quality of Life Questionnaire, functional abilities

Received: August 14, 2017; Accepted: September 21, 2017; Published: September 25, 2017 
Yingyong P (2017) Investigating the Mettapracharak (Watraikhing) vision rehabilitation outcome using the Thai version of the low vision Quality-of-Life Questionnaire (LVQOL)

cannot respond the rehabilitation procedures, severe comorbidities such as psychiatric condition, terminal disease, multiple impairments, those who declined to complete the questionnaire after rehabilitation and subjects who lost follow up. All participants gave written informed consent for participation following explanation of the nature of the study. After the demographic data collection, comprehensive eye examination and the residual vision enhancement procedures were regularly performed to utilize the remaining vision more effectively such as health education, advise and instructions in rehabilitation training communication, access service need, rehabilitation training about vision-based skills for the activity of daily living [8], prescription optical and nonoptimal low vision devices, orientation and mobility services, psychologically small group based rehabilitation support, and 3 month follow up evaluation respectively. Prior to the initiative of the present study, all field investigators were trained with the Thai version of the Low Vision Quality-of-Life Questionnaire (LVQOL) protocol [7]. It contained 6 dimensions; 22 -items (eigenvalue $>1$ including $76.9 \%$ of the total variance. Each item consisted of 1 to 5 category scales of difficulty. A higher score on LVQOL represented the better quality of life. Six domains of interest were main outcome measures (general health, difficulty with activities, social function, expectation, overall satisfaction and vision problems and responses). Before and 3-month after rehabilitation clinical information were collected in terms of LVQOL questionnaire by face to face interview.

\section{Statistical analysis}

Univariate analyses of frequencies (percent), mean, standard deviation was performed to describe patients' characteristics. $\mathrm{P}$ value $<0.05$ was considered statistically significant. LVQOL mean scores with standard deviation and $95 \%$ confidence intervals of the difference between before and 3 months after rehabilitation were analyzed by paired $t$ statistics. To achieve the outcome measurement, effect size(ES) [9] and the standardized response mean(SRM) [10] were recommended. According to cohen's benchmarks, standardized effect sizes was the standardized measure of the practical significance interpreted as large (ES $\geq 0.80)$, moderate $(0.50 \leq \mathrm{ES}<0.80)$ and small $(0.2 \leq \mathrm{ES}<0.50)$. ES was defined as the mean change score divided by the standard deviation of baseline scores and SRM was defined as the mean change score divided by the standard deviation of change for the sample. The standardized response mean (SRM) is clinically significant indicator of the rehabilitation-induced changes. The SRM values of $0.2,0.5$, and 0.8 represented small, moderate, and large changes, respectively.

\section{Results}

Among 125 visual impairment participants recruited to complete the LVQOL questionnaire before and after rehabilitation,53(42.4\%) were males and $72(57.6 \%)$ were females (Table 1). Their mean age (standard deviation) were $56 \pm 4.3$ years in males and $63 \pm 8.6$ years in females. Females were more visually impaired than males. The causes of visual impairment were glaucoma $(40.8 \%)$, age-related macular degeneration $(20.8 \%)$ and diabetic retinopathy (16.8\%) respectively (Table 2). Central visual impairment is the most common type $(80.8 \%)$ of the visual loss in Table 3. The LVQOL scores demonstrated improvements in all the domains after going low-vision rehabilitation in accordance with its statistically significance of $95 \%$ confidence interval of the different score (Table 4). There were moderately convincing evidence of the increased effect sizes and the standardized response mean changes in every domain of the LVQOL questionnaire clinically after 3 month-rehabilitation (Table 5).
Table 1. Demographic characteristics of the visual impairment patients

\begin{tabular}{|c|c|c|}
\hline & Men Number (\%) & Women Number (\%) \\
\hline Mean age (range; SD) & $56(12-82 ; \pm 4.3)$ & $63(13-88 ; \pm 8.6)$ \\
\hline $19-$-Oct & $1(1.9)$ & $2(2.8)$ \\
\hline $20-29$ & $4(7.5)$ & $5(6.9)$ \\
\hline $30-39$ & $6(11.3)$ & $6(8.3)$ \\
\hline $40-49$ & $10(18.9)$ & $12(16.7)$ \\
\hline $50-69$ & $12(22.6)$ & $17(23.6)$ \\
\hline $70-79$ & $14(26.4)$ & $21(29.2)$ \\
\hline $80-89$ & $6(11.3)$ & $9(12.5)$ \\
\hline Total & $53(100)$ & $72(100)$ \\
\hline
\end{tabular}

Table 2. Causes of visual impairment

\begin{tabular}{|l|l|}
\hline Causes & Number (\%) \\
\hline Glaucoma & $51(40.8)$ \\
\hline Age-related macular degeneration & $26(20.8)$ \\
\hline Diabetic retinopathy & $21(16.8)$ \\
\hline Retinitis pigmentosa & $13(10.4)$ \\
\hline Optic nerve diseases & $4(3.2)$ \\
\hline Corneal diseases & $5(4.0)$ \\
\hline Others* & $5(4.0)$ \\
\hline Total & $125(100)$ \\
\hline
\end{tabular}

* Includes nystagmus, cortical blindness, and hemianopia

Table 3. Type of clinical visual characteristics

\begin{tabular}{|l|l|}
\hline Type & Number (\%) \\
\hline Mainly central visual impairment & $101(80.8)$ \\
\hline Mainly peripheral visual impairment & $12(9.6)$ \\
\hline Combined visual impairment & $9(7.2)$ \\
\hline Miscellaneous* & $3(2.4)$ \\
\hline Total & $125(100)$ \\
\hline
\end{tabular}

Table 4. Mean and Standard Deviation LVQOL Scores Before and 3-Month After Rehabilitation

\begin{tabular}{|c|c|c|c|c|}
\hline Domains & $\begin{array}{c}\text { Before } \\
\text { Rehabilitation }\end{array}$ & $\begin{array}{c}\text { After } \\
\text { Rehabilitation }\end{array}$ & $\begin{array}{c}\mathbf{9 5 \%} \\
\text { Confidence } \\
\text { Interval of the } \\
\text { Difference }\end{array}$ & significance \\
\hline General health & $5.81 \pm 1.14$ & $7.98 \pm 2.33$ & $\begin{array}{c}2.18 \\
(1.71-2.64)\end{array}$ & $\mathrm{p}<0.0001$ \\
\hline $\begin{array}{c}\text { Difficulty with } \\
\text { activities }\end{array}$ & $13.34 \pm 2.63$ & $19.55 \pm 6.34$ & $\begin{array}{c}6.21 \\
(5.02-7.39)\end{array}$ & $\mathrm{p}<0.0001$ \\
\hline Social function & $7.78 \pm 1.52$ & $12.14 \pm 3.38$ & $\begin{array}{c}4.35 \\
(3.69-5.00\end{array}$ & $\mathrm{p}<0.0001$ \\
\hline $\begin{array}{c}\text { Expectation } \\
\text { Overall } \\
\text { satisfaction }\end{array}$ & $3.83 \pm 0.98$ & $5.38 \pm 1.49$ & $\begin{array}{c}1.55 \\
(1.24-1.87)\end{array}$ & $\mathrm{p}<0.0001$ \\
\hline $\begin{array}{c}\text { Vision } \\
\text { problems and } \\
\text { responses }\end{array}$ & $7.86 \pm 1.54$ & $10.62 \pm 2.49$ & $2.76(2.24-3.28)$ & $\mathrm{p}<0.0001$ \\
\hline
\end{tabular}

Statistically significant improvement at follow-up $(P<0.05)$

\section{Discussion}

In this study, females were the preponderant visually impaired gender. As many reports, they were more likely to have visual disabilities than males all over the world [11]. According to visual acuity and visual field, both factors are not absolutely to be determined all of the aspects of the visual rehabilitation [12,13]. Some research reported low vision quality of life questionnaire relating to the activity of daily living to evaluate the effect of the visual impairment rehabilitation[14-18]. To the author knowledge, there is no definitely reliable study time to follow up after visual rehabilitation may be due to worsening of eye 
Yingyong P (2017) Investigating the Mettapracharak (Watraikhing) vision rehabilitation outcome using the Thai version of the low vision Quality-of-Life Questionnaire (LVQOL)

Table 5. Effect Size (ES) and Standardized response mean(SRM) as Responsiveness Parameters 3 Months after Visual Rehabilitation ( $\mathrm{n}=125)$

\begin{tabular}{|c|c|c|}
\hline Domains & Effect size (ES) & $\begin{array}{c}\text { Standardized response } \\
\text { mean (SRM) }\end{array}$ \\
\hline General health & 0.51 & 0.53 \\
\hline Difficulty with activities & 0.54 & 0.55 \\
\hline Social function & 0.64 & 0.61 \\
\hline Expectation & 0.52 & 0.56 \\
\hline Overall satisfaction & 0.55 & 0.57 \\
\hline $\begin{array}{c}\text { Vision problems and } \\
\text { responses }\end{array}$ & 0.57 & 0.6 \\
\hline
\end{tabular}

health status, individual self-reported related magnitude of changes, variable types of activities 'difficulty, the severity of the eye diseases and the patient's expectation respectively [19]. Different time to follow up might lead to the different outcome [17]. The author prospectively used the responsiveness to validify clinically quality of life change scores after 3-month visual rehabilitation. The good responsiveness value, confirmed by the ES and SRM, resulted the good effectiveness of the visual rehabilitation outcome in all dimensions after 3-month periods. The largest of the mean score changes improvement was the difficulty with activities and the smallest one was the overall satisfactions. However, the worse results should have been reevaluated.

A strength of this study is that it is a picture of a few studies about the statistically effectiveness of the visual rehabilitation outcome in Thailand with moderately improvement.

\section{Limitation of this study}

First, this study was limited by a small sample size so these nonrandomized samples may create individual perceived data bias by chance. In order to confirm these findings, longer follow up for visual needs and demands of visual rehabilitation and a large sample sizes are essential for the visual impairment patients to enhance their functional disabilities and independence. Second, the research process may depend on many confounding factors such as age, sex, different ophthalmologic conditions, severity and types of visual impairment, living condition, socioeconomic status and the time onset of the eye conditions. Third, all the missing data might be different from these enrolled participants.

\section{Conclusion}

This present study showed the statistically moderate improvement after 3-month visual rehabilitation in Mettapracharak(Watraikhing) low vision service. Nevertheless, more low vision rehabilitation practitioners should be trained to provide the services as the increasing numbers of the visually impaired patients. Finally, multidisciplinary holistic approaches are necessary to overcome many kinds of eye diseases and their progression in the long-term periods.

\section{Acknowledgement}

The author wishes to thank the Director of the Mettapracharak Eye Center, Nakhonpathom (Dr. Pannet Pangputhipong) for his valuable participation. The author is grateful to all other members of the study team who made this study possible.

\section{References}

1. Global data on visual impairments 2010 - World Health

2. Wongwetsawat S. Report of the third national evaluation of prevention of blindness program in Thailand. Lampang. Thailand: Kijseri 1997.
3. Jenchitr W. The Fourth National Survey of Blindness, Low Vision and Major Eye Diseases in Thailand The first Visual Impairment Project in Thailand-TVIP 2006-2007).

4. Hinds A, Sinclair A, Park J, Suttie A, Paterson H, et al. (2003) Impact of an interdisciplinary low vision service on the quality of life of low vision patients. $\mathrm{BrJ}$ Ophthalmol 87: 1391-1396. [Crossref]

5. Stelmack JA, Stelmack TR, Massof RW (2002) Measuring low-vision rehabilitation outcomes with the NEI VFQ-25. Invest Ophthalmol Vis Sci 43: 2859-2868. [Crossref]

6. Wolffsohn JS, Cochrane AL (2000) Design of the low vision quality-of-life questionnaire (LVQOL) and measuring the outcome of low-vision rehabilitation. Am J Ophthalmol 130: 793-802. [Crossref]

7. Yingyong P (2007) Evaluation of the Thai, Low Vision Quality-of-Life Questionnaire (LVQOL). J Med Assoc Thai 90: 2658-2661. [Crossref]

8. Stelmack JA, Szlyk JP, Stelmack TR, Demers-Turco P, Williams RT, et al. (2006) Measuring outcomes of vision rehabilitation with the Veterans Affairs Low Vision Visual Functioning Questionnaire. Invest Ophthalmol Vis Sci 47: 3253-3261. [Crossref]

9. Cohen J. Statistical power analysis for the behavioural sciences. rev. ed. New York Academic Press; 1977.

10. Crosby RD, Kolotkin RL, Williams GR (2004) An integrated method to determine meaningful changes in health-related quality of life. J Clin Epidemiol 57: 1153-1160. [Crossref]

11. Courtright P, Bassett K (2003) Gender and blindness: eye disease and the use of eye care services. Community Eye Health 16: 11-12. [Crossref]

12. Scott IU, Schein OD, West S, Bandeen-Roche K, Enger C, et al. (1994) Functional status and quality of life measurement among ophthalmic patients. Arch Ophthalmol 112: 329-335. [Crossref]

13. Sloane ME, Ball K, Owsley C, Bruni JR, Roenker DL. (1992) The visual activities questionnaire:developing an instrument for assessing problems in everyday visual tasks. Tech Dig Noninvasive Assess Vis Sys 1: 26-9.

14. Stelmack J (2001) Quality of life of low-vision patients and outcomes of low-vision rehabilitation. Optom Vis Sci 78: 335-342. [Crossref]

15. Mc Horney CA, Tarlov A. (1995) Individual-patient monitoring in clinical practice: Are available health status measures adequate? Qua Life Res 4: 293-307. [Crossref]

16. Stelmack JA, Moran D, Dean D, Massof RW (2007) Short- and long-term effects of an intensive inpatient vision rehabilitation program. Arch Phys Med Rehabil 88: 691-695. [Crossref]

17. Kuyk T, Liu L, Elliott JL, Grubbs HE, Owsley C, et al. (2008) Health-related quality of life following blind rehabilitation. Qual Life Res 17: 497-507. [Crossref]

18. Lamoureux EL, Pallant JF, Pesudovs K, Rees G, Hassell JB, et al. (2007) The effectiveness of low-vision rehabilitation on participation in daily living and quality of life. Invest Ophthalmol Vis Sci 48: 1476-1482. [Crossref]

19. Wolffsohn JS, Cochrane AL, Watt NA (2000) Implementation methods for vision related quality of life questionnaires. Br J Ophthalmol 84: 1035-1040. [Crossref]

Copyright: (C2017 Yingyong P. This is an open-access article distributed under the terms of the Creative Commons Attribution License, which permits unrestricted use, distribution, and reproduction in any medium, provided the original author and source are credited. 